\title{
Améliorer les conditions de vie dans les quartiers défavorisés en France
}

Écrit par : Nicola Brandt

Dernière mise à jour : 19 juin 2018

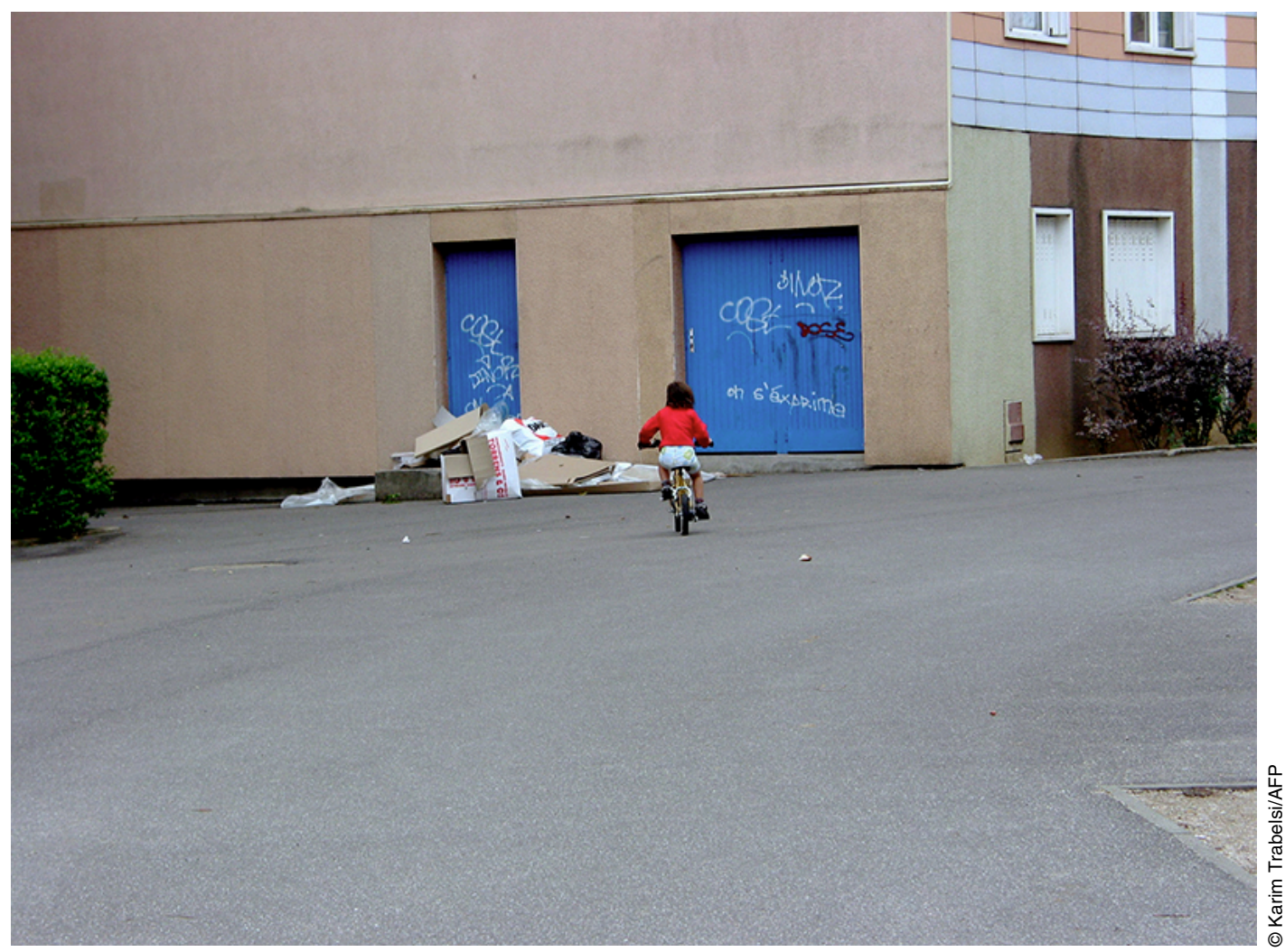

Si dans l'ensemble, la pauvreté globale est relativement faible en France, elle peut être fortement concentrée au niveau de certains quartiers, où jusqu'à $\mathbf{4 0} \%$ des ménages peuvent vivre en dessous du seuil de pauvreté. Le chômage y est élevé, les élèves sont en difficulté, les logements et les infrastructures urbaines en mauvais état, sans compter le manque d'entreprises, de services publics et privés et d'aménagements au plan local. Les pouvoirs publics déploient dans ces zones des mesures ciblées en faveur de l'éducation, de l'emploi, de la sécurité et des entreprises.

Des politiques visant à promouvoir la mixité sociale sont aussi mises en œuvre : dans les zones sous tension, les municipalités doivent ainsi disposer d'un quota 
d'au moins $25 \%$ de logements sociaux, sous peine de se voir infliger des amendes. Le Programme national pour la rénovation urbaine est un programme d'envergure qui vise à attirer les habitants des quartiers plus aisés vers les quartiers défavorisés où le parc de logements sociaux délabrés a laissé la place à des unités de plus petite taille, mêlant habitat social, logements locatifs privés et logements occupés par leurs propriétaires. Ces politiques sont fondées sur l'idée que la concentration géographique de la pauvreté est un facteur de renforcement et de reproduction des handicaps économiques et sociaux. Aux États-Unis, on observe d'ailleurs que les enfants qui quittent des quartiers où la pauvreté se concentre accèdent à des niveaux d'éducation et de revenu plus élevés que ceux qui y restent.

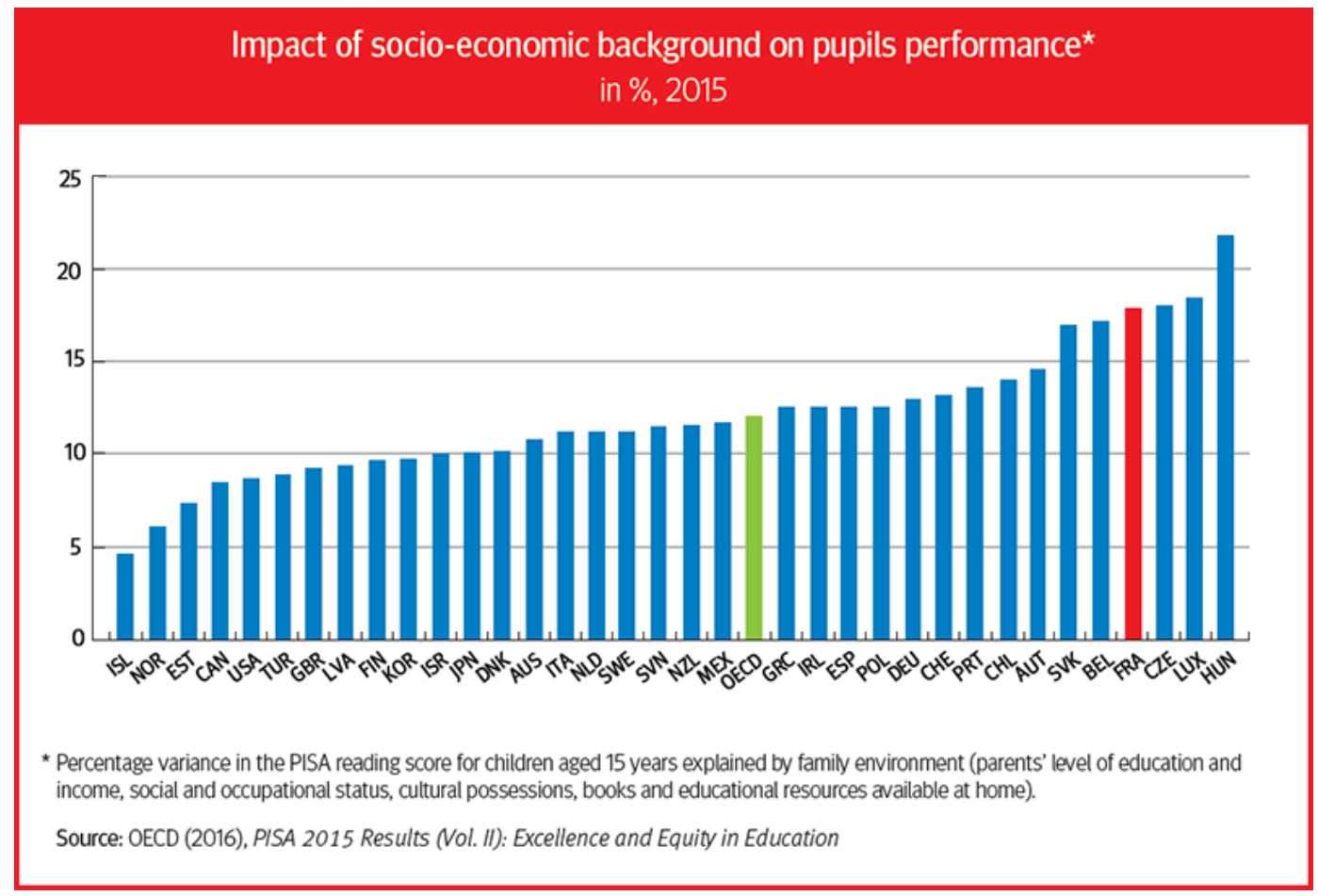

Bien que ces résultats ne soient pas nécessairement transposables dans le contexte français, où la pauvreté est globalement beaucoup plus faible et le système de sécurité sociale plus développé qu'aux États-Unis, il apparaît clairement que les habitants des quartiers pauvres en France pâtissent d'un handicap social plus marqué. Le risque de chômage pour ces populations est presque deux fois plus élevé que pour les personnes ayant des profils comparables, mais vivant dans des quartiers plus favorisés. Les études montrent que ce phénomène est en partie une conséquence de la discrimination : les personnes portant des patronymes à consonance étrangère ou dont l'adresse indique qu'ils habitent dans des quartiers défavorisés ont moins de chances de décrocher des entretiens d'embauche. Une autre difficulté tient au fait que de nombreux quartiers défavorisés sont isolés et mal desservis par les infrastructures et services de transport. Ce problème est particulièrement aigu en 
dehors des heures de pointe, et touche particulièrement les travailleurs peu qualifiés qui sont également moins susceptibles d'être titulaires d'un permis de conduire ou de posséder une voiture. Les auteurs de l'Étude économique de l'OCDE sur la France, publiée en 2017, jugent nécessaire de mettre en œuvre des politiques actives de lutte contre la discrimination. Ces mesures peuvent recouvrir des campagnes de sensibilisation auprès des recruteurs, des activités de tutorat et d'accompagnement auprès des demandeurs d'emploi et des placements directs auprès d'employeurs potentiels, système qui a bien fonctionné pour les diplômés de l'université.

Le programme de rénovation urbaine a permis d'améliorer les infrastructures et de nombreux habitants sont satisfaits des résultats obtenus, mais l'impact sur la mixité sociale n'est pas établi. Comme cela a été le cas dans d'autres pays, de nombreuses personnes qui habitaient dans des bâtiments démolis pour faire place à des logements mixtes ont finalement été relogées dans d'autres quartiers tout aussi déshérités. De plus, la mixité de l'habitat ne s'est pas accompagnée d'un renforcement des interactions sociales.

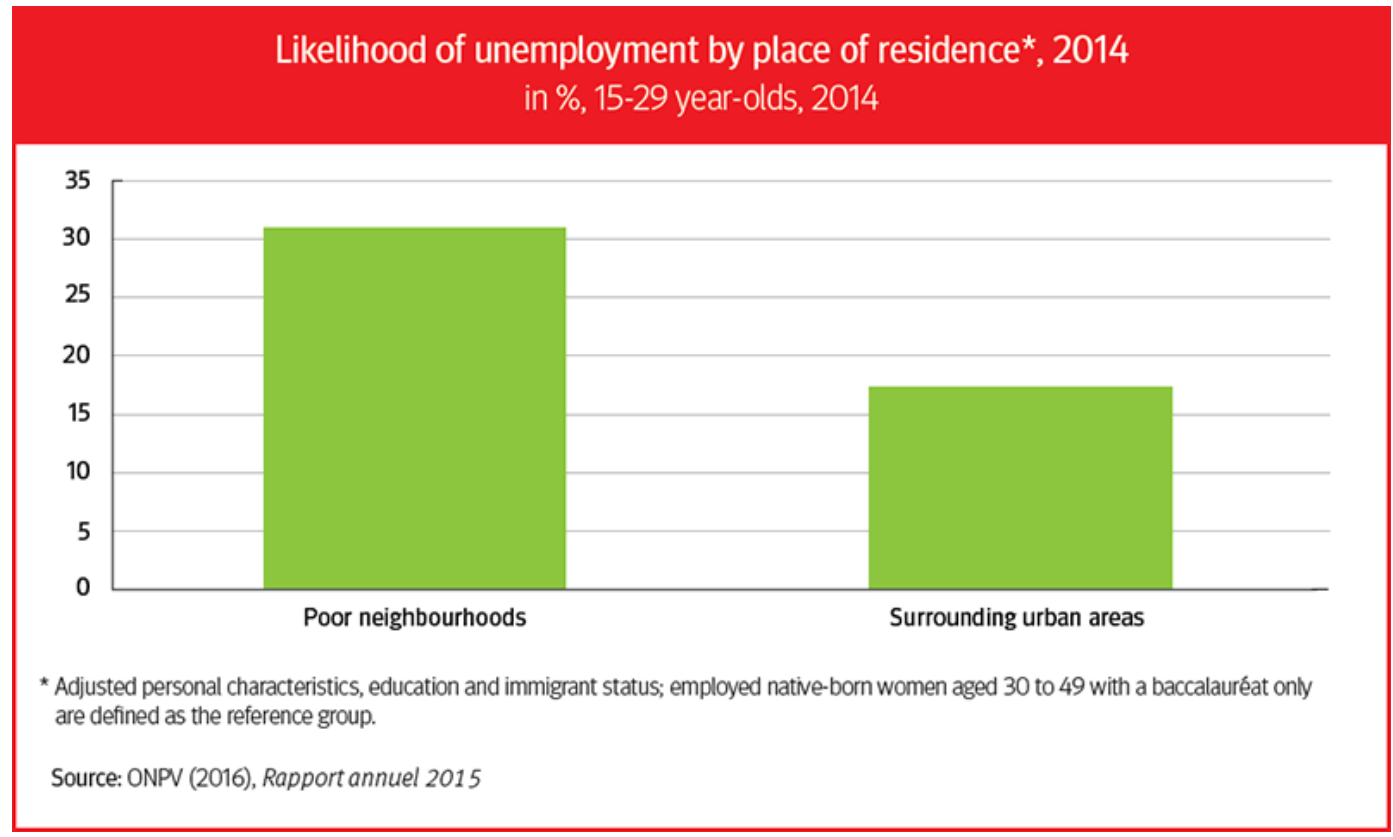

Alors que le gouvernement prévoit d'étendre le programme, les auteurs de l'Étude économique recommandent une meilleure coordination avec les politiques sociales et d'emploi. La consultation des habitants sur les projets de rénovation pourrait également constituer un point de départ pour une formation aux compétences de base et en langue. Les travaux de construction et de rénovation pourraient être l'occasion de proposer des formations en apprentissage dans le secteur du bâtiment. La consultation doit absolument être renforcée et les conseils citoyens mis en place en 2014 pour aider à l'élaboration de documents de stratégie de développement économique et social des quartiers déshérités constituent un premier pas dans cette direction. Associer étroitement les habitants à la 
conception et à la mise en œuvre de projets de rénovation est une expérience qui s'est révélée fructueuse en Allemagne, où les résidents réhabilitent généralement eux-mêmes leurs logements. Aux États-Unis, les habitants de bâtiments voués à la démolition ont souvent mal vécu le relogement forcé. Ils ont désormais le droit de réintégrer leur logement une fois la reconstruction achevée.

Depuis plus de 30 ans, la France a mis en place des programmes d'éducation prioritaire permettant d'allouer davantage de ressources aux établissements scolaires accueillant des élèves de milieux défavorisés. Cependant, la mesure de l'influence de la situation socioéconomique des familles sur les résultats scolaires reste l'une des plus élevée de l'OCDE. Selon l'Étude économique sur la France de 2017, les mesures d'accompagnement individuel des élèves en difficulté pâtissent d'un accès insuffisant des enseignants à une formation initiale et continue de qualité en pédagogie. Il faut faire davantage pour offrir des perspectives de rémunération et de carrière attrayantes aux enseignants qui travaillent dans des établissements comptant une forte population d'enfants défavorisés. Si la prime octroyée aux enseignants du réseau d'éducation prioritaire a récemment été relevée, elle reste trop faible pour stabiliser les équipes éducatives. Effet pervers de la mesure, les avantages dont bénéficient les enseignants affectés à ces établissements les encouragent en fait à en partir plus rapidement.

@L'Observateur de l'OCDE n³11 T3 2017

\section{Références}

Bunel, M., Y. L'Horty and P. Petit (2016), « Discrimination based on place of residence and access to employment », Urban Studies, Vol. 53, No. 2, pp. 267-86. http://journals.sagepub.com/doi/abs/10.1177/0042098014563470

Chetty, R. and N. Hendren (2015), " The impacts of neighborhoods on intergenerational mobility: childhood exposure effects and county-level estimates ». https:// scholar.harvard.edu/files/hendren/files/nbhds_paper.pdf

Chetty, R., N. Hendren and L. Katz (2016), « The Effects of Exposure to Better Neighborhoods on Children: New Evidence from the Moving to Opportunity Experiment », American Economic Review, Vol. 106, No. 4, pp. 855-902, April. https://www.aeaweb.org/articles?id=10.1257/aer.20150572

Kirszbaum, T. (2013), « Rénovation urbaine et équité sociale: Choice Neighborhoods aux Etats-Unis ", [Rapport de recherche] ISP; CNRS; Commissariat général à la stratégie et à la prospective; secretariat général du Comité interministériel des villes. https://halshs.archives-ouvertes.fr/ halshs-01265144/document 